\title{
Use of a Modified Tibial Plateau Levelling Osteotomy with Double Cut and Medial Crescentic Closing Wedge Osteotomy to Treat Dogs with Cranial Cruciate Ligament Rupture and Tibial Valgus Deformity
}

\author{
Luca Vezzoni ${ }^{1}$ Sara Bazzo ${ }^{2}$ Silvia Boiocchi ${ }^{1} \quad$ Aldo Vezzoni $^{1}$ \\ ${ }^{1}$ Surgical Department, Clinica Veterinaria Vezzoni, Cremona, Italy \\ 2 Surgical Department, Clinica Veterinaria Malpensa, Samarate, Italy \\ Address for correspondence Luca Vezzoni, DVM, Dipl ECVS, Clinica \\ Veterinaria Vezzoni, Via Massarotti 60/A, 26100, Cremona, Italy \\ (e-mail: lucavezzoni@me.com).
}

Vet Comp Orthop Traumatol 2020;33:59-65.

\begin{abstract}
Objective The aim of this study was to report efficacy of a modified tibial plateau levelling osteotomy (TPLO) with double cut and medial crescentic closing wedge osteotomy (TPLO/MCCWO) to treat dogs with cranial cruciate ligament rupture and concurrent tibial valgus.

Study Design This study was a cases series.

Materials and Methods Medical records of dogs that had TPLO with medial crescentic closing wedge osteotomy were reviewed. Data collected included signalment, body weight, pre- and postoperative tibial valgus angle, tibial plateau angle (TPA), surgical planning, corrective osteotomy technique, method of fixation, complications, and length of time to radiographic healing.

Results Fifty-two surgical procedures performed in 45 dogs (7 bilateral) were included in the study. Mean age at surgery was 54 months, and body weight ranged from 5 to $63 \mathrm{~kg}$ (mean: $36.5 \mathrm{~kg}$ ). Mean pre- and postoperative mechanical medial proximal tibial angle were $101^{\circ}\left(98^{\circ}-107^{\circ}\right)$ and $92.80^{\circ}\left(88^{\circ}-97^{\circ}\right)$ respectively. The mean pre- and postoperative TPA were $27.80^{\circ}\left(16-35^{\circ}\right)$ and $6.50^{\circ}\left(3-11^{\circ}\right)$ respectively. Intraoperative complications occurred in two stifles: in one stifle over-rotation of the

Keywords

- canine

- cranial cruciate ligament rupture

- tibial plateau levelling osteotomy

- tibial valgus proximal tibial segment resulted in a TPA of $-8^{\circ}$, with immediate revision to a $5^{\circ} \mathrm{TPA}$; in the second stifle a fissure of the lateral tibial cortex developed during insertion of a screw and required adjunctive fixation. No postoperative complications were recorded and all osteotomies healed uneventfully.

Conclusions Tibial plateau levelling osteotomy/medial crescentic closing wedge osteotomy is an effective treatment for dogs with cranial cruciate ligament rupture and tibial valgus allowing accurate correction of the tibial deformity with a low complication rate.
\end{abstract}

\section{Introduction}

Tibial plateau levelling osteotomy (TPLO) is a popular procedure for the treatment of cranial cruciate ligament deficiency and has proved to be reliable and effective in restoring stifle function..$^{1-3}$ In some dogs, cranial cruciate ligament deficiency may occur with proximal tibial deformities in the frontal plane (tibial varus or tibial valgus). ${ }^{4}$ Valgus deformity of the proximal tibia has been shown to result in an abnormal load on the articular cartilage, leading to erosion of the cartilage of the received

December 27, 2018

accepted after revision

July 20, 2019

published online

November 22, 2019
(C) 2020 Georg Thieme Verlag KG Stuttgart . New York
DOI https://doi.org/ 10.1055/s-0039-1700565. ISSN 0932-0814. 
lateral femorotibial compartment. ${ }^{5,6}$ The severity of angular deformity of the knee in humans is correlated with the severity of osteoarthritis as well as the degree of anterior cruciate ligament degeneration. ${ }^{7,8}$

For these reasons, valgus deformity should be assessed at the time of cranial cruciate ligament rupture treatment. A modification of the original TPLO technique was described previously to simultaneously treat cranial cruciate ligament rupture and proximal tibial deformity by combining levelling osteotomy with a transverse tibial osteotomy just distal to the TPLO. ${ }^{4}$ This technique is effective in correcting proximal tibial deformities and levelling the tibial plateau angle (TPA). However, it is technically demanding, and compared with a standard TPLO requires the use of a reinforced plate in combination with a double tension band wire. ${ }^{4}$

Fitzpatrick and colleagues proposed a modified TPLO with medial crescentic opening wedge osteotomy to treat tibial deformities on the frontal plane associated with cranial cruciate ligament rupture, but this technique assesses only varus deformities and cannot be applied to valgus deformities. ${ }^{9}$

Barclay Slocum described an alternative to this method consisting of a modified TPLO with double cut and medial crescentic closing wedge osteotomy (MCCWO) (Seminar titled TPLO for Cranial Cruciate Ligament Repair; Slocum Enterprises Inc., November 1997, Dresden, Germany). To our knowledge, the results of this technique have not been reported.

\section{Objective}

The objective of this retrospective study was to determine the efficacy of TPLO with MCCWO for correction of angular deformity and levelling of TPA in dogs with cranial cruciate ligament rupture and concurrent tibial valgus deformity.

\section{Materials and Methods}

Medical records of dogs treated with TPLO/MCCWO between April 2007 and April 2018 at the Vezzoni Veterinary Hospital were reviewed. Data retrieved included signalment, weight (kilograms), clinical findings, gross assessment of tibial deformity (valgus, tibial torsion, or some combination of these), cranial cruciate ligament integrity and patellar stability, preand postoperative TPA, mechanical medial proximal tibial angle (mMPTA), mechanical medial distal tibial angle (mMDTA), tibial torsion, concurrent patellar luxation and femoral deformities, intraoperative condition of cruciate ligaments, menisci and articular cartilage, fixation implants used, concurrent surgical procedures and intraoperative and postoperative complications encountered. Complications were considered major if additional surgery or medical treatment was required, as previously described; minor complications were those that did not require additional surgery or medical treatment. ${ }^{10}$ Inclusion criteria were lameness attributable to partial or complete rupture of the cranial cruciate ligament, the presence of a preoperative $\mathrm{MMPTA}>97^{\circ}$ and clinical and radiographic follow-up examination a minimum of 6 months postoperatively.

\section{Surgical Planning}

Craniocaudal and mediolateral radiographs of the tibia, including stifle and talocrural joints, were obtained for both hind limbs. Radiographic surgical planning was done using scaled digital radiographic images and a commercially available PACS software (AGFA NX viewer, Agfa Health Care NV B-2640 Mortsel, Belgium) (-Fig. 1). On the mediolateral view, the preoperative TPA was determined according to standard guidelines. ${ }^{1,11-13}$ The osteotomy was planned by placing a TPLO saw blade template of appropriate size on the scaled tibial image so that the blade was centred over a point dividing the intercondylar tubercles. The following three reference points were recorded: D1, the distance from the perpendicular cranial straight edge of the tibial crest originating at the most cranioproximal point of the tibial tuberosity to the intended osteotomy; D2, the distance from the most cranioproximal point of the tibial tuberosity and extending to the point where the intended tibial osteotomy transected the cranial tibial subchondral bone; and D3, the distance from the subchondral bone at the most caudal margin of the tibial plateau to the point where the intended tibial osteotomy transected the caudal tibial cortex (-Fig. 1A). The intended rotation achieved with TPLO was then calculated using a standard TPLO reference chart to obtain a target postoperative TPA of $6^{\circ} \cdot{ }^{14,15}$ On the caudocranial view, the MMPTA was determined as described previously $^{16}$; this angle was compared with the published reference angle $\left(93.30^{\circ} \pm 1.781^{\circ}\right)$. The MMPTA was measured also on the contralateral limb; this value was considered normal and used as a reference point if it was within the $95 \%$ confidence interval as reported previously. ${ }^{16}$ In cases of unilateral deformity, the amount of correction required was calculated with the following formula: correction angle $=$ affected tibia MMPTA - contralateral tibia MMPTA. In cases of bilateral deformities, the following formula was used: correction angle $=$ affected tibia mMPTA $-93^{\circ}$. Distance D3 was then reproduced and marked on a digital radiograph, and a wedge of the angle intended to correct the MMPTA was drawn at the distal aspect of this distance, measuring the width of the wedge on the medial cortex of the tibia (-Fig. 1B). On the same radiographic view, the mMDTA was measured as described. ${ }^{17}$

\section{Surgical Technique}

Dogs were placed under general anaesthesia and positioned in dorsal recumbency and the affected limb was aseptically prepared for surgery in a hanging position. A standard medial surgical approach to the stifle and proximal tibia was used. A medial parapatellar mini-arthrotomy was done to inspect the cruciate ligaments, medial and lateral menisci and the visible area of femoral cartilage. When meniscal lesions were identified, partial meniscectomy of the damaged meniscal segment was performed. A standard TPLO jig (Slocum Enterprises Small and Large TPLO jig, Slocum Enterprises, Eugene, Oregon, United States; Synthes Large TPLO Jig, DePuy Synthes Vet, West Chester, Pennsylvania, United States) was assembled with the proximal pin placed 3 to $5 \mathrm{~mm}$ distal to the tibial plateau, just caudal to the medial collateral ligament in a 


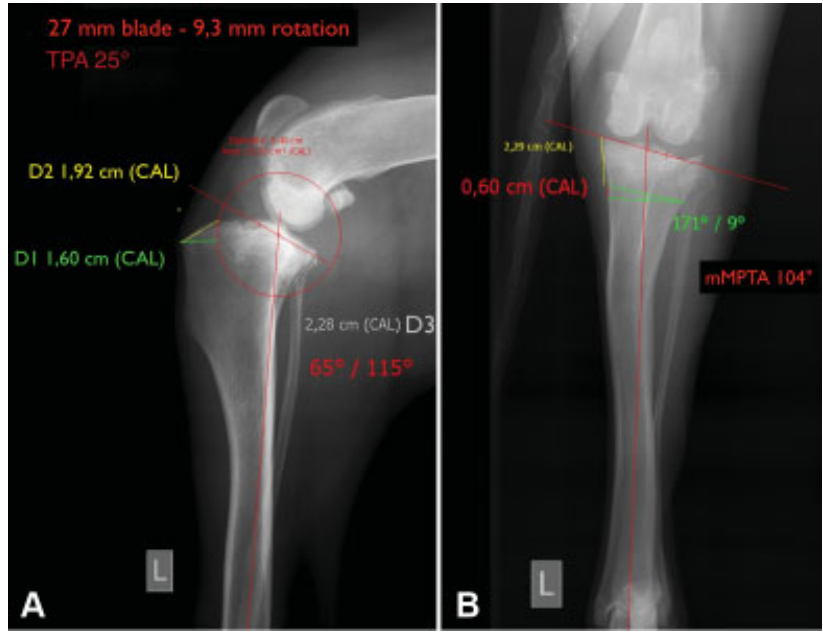

Fig. 1 Radiographic planning for tibial plateau levelling osteotomy/medial crescentic closing wedge osteotomy. On the mediolateral view, tibial plateau angle (TPA) is measured and the position and size of the osteotomy are planned based on three measurements (D1, D2 and D3), which are used as a reference during surgery $(A)$. On the caudocranial view, the mechanical medial proximal tibial angle (mMPTA) is determined, distance D3 is then drawn and a wedge of the angle intended to correct mMPTA to the desired value is drawn at the distal aspect of D3, measuring the width of the wedge on the medial cortex of the tibia (B). direction that was parallel to the proximal tibial joint surface and perpendicular to the patellar ligament (-Fig. 2A). The proximal tibial valgus deformity resulted in displacement of the distal aspect of the jig away from the medial tibial cortex (-Fig. 2B). The distal jig pin was inserted through the distal jig hole in a direction parallel to the proximal one and driven through both cortices. Once the jig had been placed, the lateral and caudal soft tissues were packed off with wet sponges placed between the tibia and the cranial tibial muscle laterally and the popliteal muscle caudally.

The osteotomy position was then marked using distances D1, D2 and D3. A standard TPLO osteotomy was started using the selected TPLO saw blade (Synthes crescentic TPLO blades, DePuy Synthes Vet, West Chester, Pennsylvania, United States), keeping the blade parallel to the distal jig pin, and was partially completed by cutting entirely through the cis cortex but not the trans cortex (-Fig. 2C). Before finishing the osteotomy, the calculated amount of TPLO rotation was marked on the medial cortex using an osteotome, with the distal mark translated as a radial line across the osteotomy to the distal segment, making sure to extend it distally over the planned width of the closing wedge osteotomy. The width of the closing wedge osteotomy was then marked with an osteotome at the level of D3 (-Fig. 2D). Starting from this

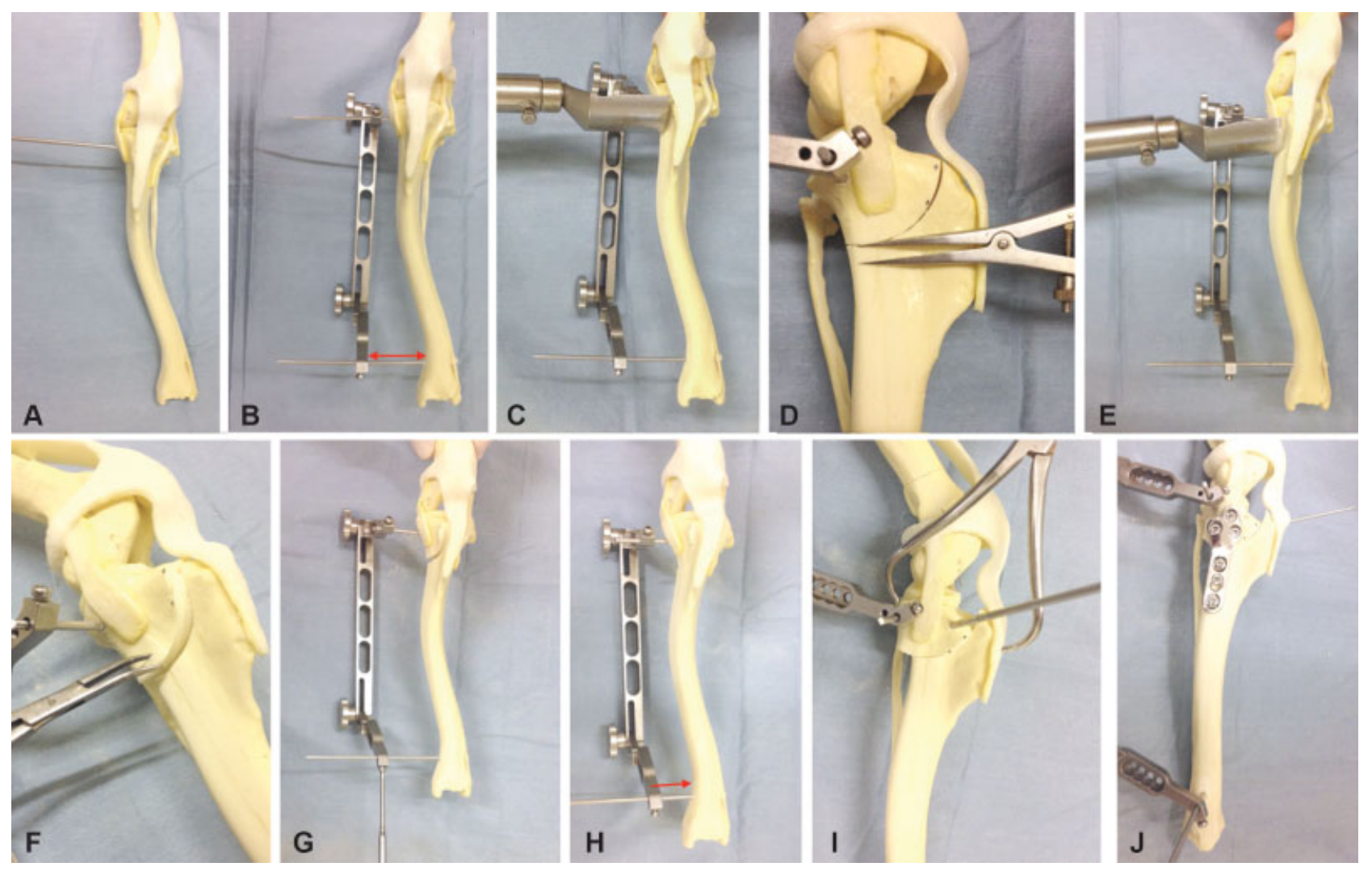

Fig. 2 A bone model showing the position of the proximal jig pin parallel to the proximal tibial joint surface and perpendicular to the patellar ligament (A). Because of the proximal tibial valgus, there is increased distance between the distal part of the jig and the medial tibial cortex (B). The saw blade is kept parallel to the distal jig pin to start the standard tibial plateau levelling osteotomy (TPLO) osteotomy, which is then partially completed by cutting the cis cortex (C). Before completion of the osteotomy, the width of the closing wedge osteotomy is marked with an osteotome at the level of D3 (D) and the second cut is made distal to the first, keeping the blade at an angle to the jig pin that corresponds to the planned correction (E). After completion of both osteotomies, the resulting bone wedge is removed (F) creating a gap at the medial cortex. The distal jig screw is then loosened $(\mathbf{G})$, and the distal aspect of the jig is moved towards the tibial diaphysis to close the medial osteotomy gap ( $\mathrm{H}$ ). The proximal tibial segment is then rotated $(\mathrm{I})$ and the osteotomy stabilized with a standard TPLO plate (J). 

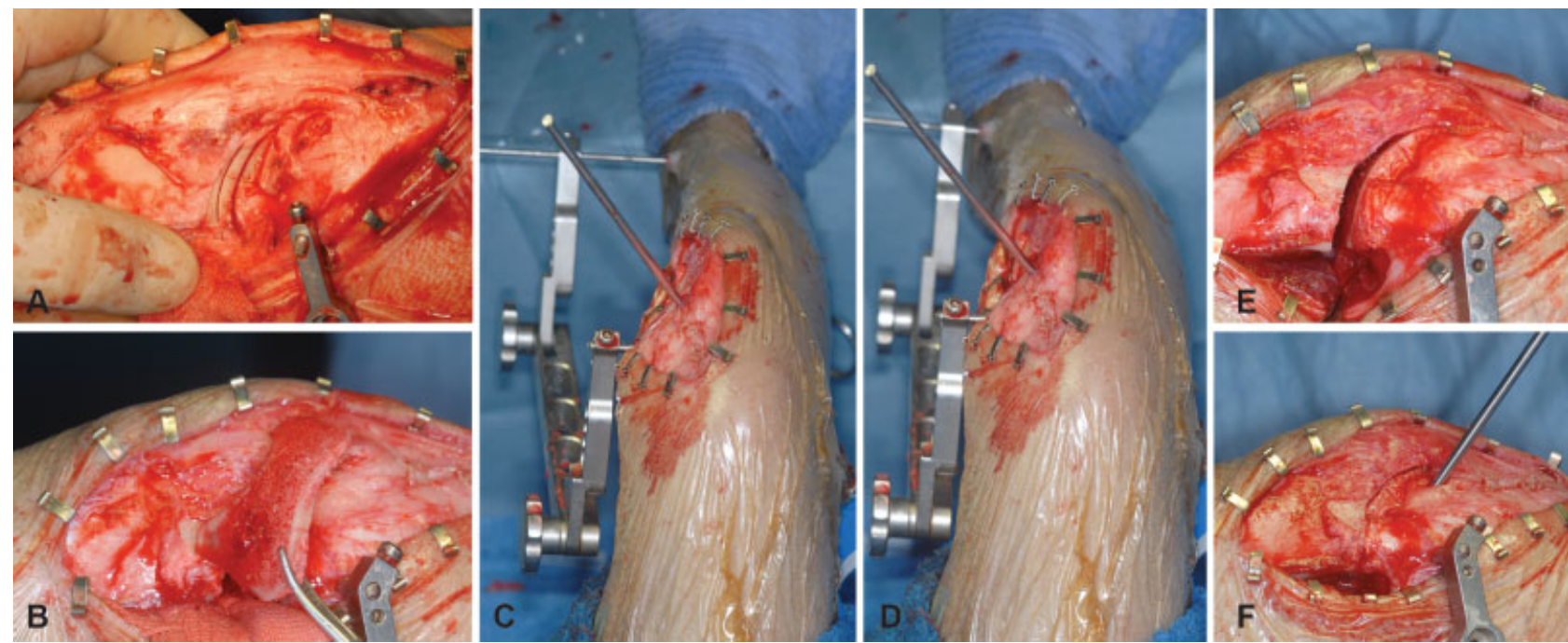

Fig. 3 Intraoperative images of the case shown in - Fig. 1. The two osteotomy lines are visible on the medial tibial cortex (A). Removal of the bone wedge after completion of the two osteotomies (B). The distal part of the jig, which is displaced from the medial tibial cortex (C), is moved laterally towards the limb (D) and the remaining gap (E) is obliterated (F).
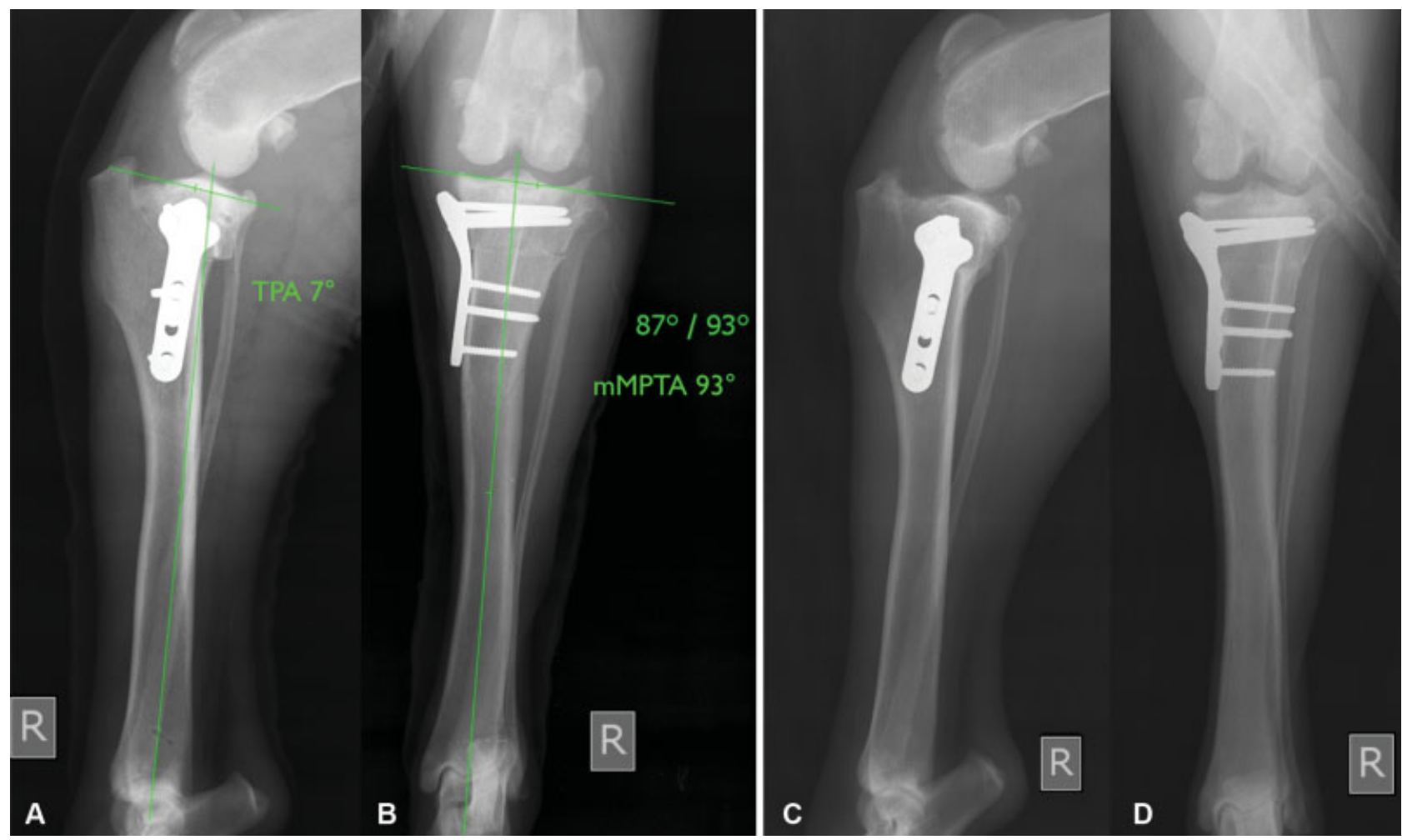

Fig. 4 Radiographs of the limb shown in - Figs. 1 and $\mathbf{3}$ taken immediately postoperatively and 2 months later. Postoperative radiographs (A, B) show levelling of the tibial plateau angle (TPA) to $7^{\circ}$ and correct alignment of the proximal tibia in the frontal plane. Radiographs taken 2 months after surgery $(C, D)$ show complete bone healing. mMPTA, mechanical medial proximal tibial angle.

mark, a second cut was made distal to the first, touching the D2 landmark and keeping the blade at an angle to the jig pin corresponding to the planned correction (-Fig. 2E). A partial elevation of the cranial tibial muscle on the lateral aspect of the tibia was made to allow visual estimation of the exit point of the saw blade to have the two osteotomy lines meeting at the apex on the lateral side. After completion of both osteotomies, the resulting bone wedge was removed ( - Fig. 2F), leaving a gap on the medial cortex ( - Fig. 3 ).

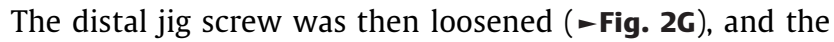
distal aspect of the jig was moved towards the tibial diaphysis to close the medial osteotomy gap created by the double cut ( - Fig. 2 H). The proximal tibial segment was then rotated to the planned amount to obtain a target postoperative TPA of $6^{\circ}$ (-Fig. 2I). The osteotomy line was compressed with pointed reduction forceps, and the proximal tibial segment was secured with a temporary Kirschner wire, placed approximately $3 \mathrm{~mm}$ proximal to the insertion point of patellar 
ligament on the tibial tuberosity and driven to the proximal tibial segment. The osteotomy was stabilized with a standard TPLO plate ( - Fig. 2J). The TPLO jig and pins were removed and the tissue layers closed in routine fashion. Postoperative mediolateral and craniocaudal radiographs were assessed for correct positioning of the implants and measurement of the TPA, MMPTA and mMDTA ( - Fig. 4A, B). A modified Robert Jones bandage was applied for 24 to 48 hours and restriction of physical activity was recommended for 6 weeks. Skin suture removal was done 2 weeks postoperatively. After discharge the dogs received amoxicillin/clavulanic acid $(20 \mathrm{mg} / \mathrm{kg}$ orally thrice daily) for 5 days and meloxicam $(0.05 \mathrm{mg} / \mathrm{kg}$ SID, PO) for 1 week and then on alternate days for another 2 weeks.

\section{Clinical and Radiographic Follow-Up}

Clinical and radiographic assessments were done 8 to 10 weeks and 6 months postoperatively. At each examination, lameness was evaluated and signs of pain or discomfort during manipulation of the stifle joint were recorded. Mediolateral and caudocranial radiographic views were obtained to assess bone healing of the osteotomy and to identify complications or abnormalities associated with the implants. Bone healing was retrospectively evaluated using an established scoring system, which defined union as fusion of the fracture line, the presence of bridging callus or the disappearance of the fracture lines seen on each radiographic view ( - Fig. 4C, D). Scores were $1=$ poor union with $<25 \%$ healing and no evidence of callus, $2=$ fair union with 25 to $50 \%$ healing, $3=$ good union with $>50$ to $75 \%$ healing and $4=$ excellent union with $>75 \%$ healing. ${ }^{17}$

\section{Statistical Methods}

Commercially available software (SPSS 22: IBM Corp, Armonk, New York, United States) was used for statistical analysis. To evaluate the accuracy of correction of alignment, a paired $t$-test was used to compare the planned and effective changes in MMPTA and TPA. A paired $t$-test was used to compare preoperative and postoperative mMDTA to evaluate the effect of the procedure on alignment of the distal tibia. Statistical significance was set at $p \leq 0.05$.

\section{Results}

Fifty-two TPLO/MCCWO procedures were performed on 45 dogs (7 bilateral) by three different surgeons (L.V., A.V., Sa. B.). Mean age at surgery was 54 months (range: $12-120$ months), and body weight ranged from 5 to $63 \mathrm{~kg}$ (mean: $36.5 \mathrm{~kg}$ ). Several breeds were included but the German Shepherd dog was most common (13 cases), followed by mixed-breed ( 7 cases) and Dobermann Pinscher (6 cases). Forty-three out of 52 (82.7\%) operations were done in dogs $>15 \mathrm{~kg}$ body weight. Nine of $52(17.3 \%)$ operations were done in dogs $<15 \mathrm{~kg}$ body weight, and the smallest dog weighed $5 \mathrm{~kg}$. Cranial cruciate ligament rupture was identified in all stifles: complete rupture in 40 stifles and partial rupture in 12 . Lesions were identified in the medial meniscus in 11 stifles, in the lateral meniscus in 3 stifles and in both medial and lateral menisci in 1 stifle. Meniscal release was performed in 22 stifles with acute cranial cruciate ligament rupture and marked instability. Cartilage erosion was seen on the lateral femoral condyle in five stifles, and osteochondritis dissecans of the lateral femoral condyle in two stifles. Three stifles had concurrent patellar luxation, which was grade II medial patellar luxation in two and grade III lateral patellar luxation in one. Concurrent surgical procedures included block trochleoplasty in two stifles with medial patellar luxation, block trochleoplasty and distal femoral corrective osteotomy in the one stifle with lateral patellar luxation associated with distal femoral valgus deformity and autogenous osteochondral graft in the two stifles with osteochondritis dissecans of the lateral femoral condyle. For small dogs, the $10 \mathrm{~mm}$ saw blade was used in two cases and the $12 \mathrm{~mm}$ saw blade in five cases. The manufacturer and size of the implants varied (-Table $\mathbf{1}$ ). Mean pre- and postoperative mMPTA were $101^{\circ}\left(98^{\circ}-107^{\circ}\right)$ and $92.80^{\circ}$ $\left(88-97^{\circ}\right)$ respectively. The mean change in MMPTA was $7.96^{\circ}$ (standard deviation [SD]: 2.27 ), and the mean planned correction angle was $7.92^{\circ}$ (SD: 2.57). The difference between accomplished and planned changes in MMPTA was not significant $(p 0.9031)$. The mean preoperative mMDTA was $95.48^{\circ}\left(88^{\circ}-101^{\circ}\right)$ (SD: 2.41 ), and the mean postoperative mMDTA was $95.38^{\circ}\left(88-100^{\circ}\right)$ (SD: 2.40$)$. The difference between the preoperative and postoperative mMDTA was not significant $(p$ 0.1996). The mean pre- and postoperative TPA were $27.80^{\circ}\left(16-35^{\circ}\right)$ and $6.50^{\circ}\left(3-11^{\circ}\right)$ respectively. The mean change in TPA was $21.35^{\circ}$ (SD: 4.35) and the mean planned tibial plateau correction angle was $21.83^{\circ}$ (SD: 4.00).

Table 1 Fixation implants used: manufacturer, screws diameter and type

\begin{tabular}{|l|l|l|l|}
\hline Manufacturer & $\begin{array}{l}\text { Screw } \\
\text { diameter } \\
(\mathbf{m m})\end{array}$ & $\begin{array}{l}\text { Locking } \\
\text { plate } \\
\text { (yes/no) }\end{array}$ & $\begin{array}{l}\text { Number } \\
\text { of cases }\end{array}$ \\
\hline Synthes $^{\text {a }}$ & 2.4 & Yes & 2 \\
\hline Synthes $^{\text {a }}$ & 2.7 & Yes & 3 \\
\hline Synthes $^{\text {a }}$ & 3.5 & Yes & 17 \\
\hline Synthes Broad $^{\text {a }}$ & 3.5 & Yes & 8 \\
\hline Fixin $^{\text {b }}$ & 2.5 & Yes & 7 \\
\hline Fixin $^{\text {b }}$ & 3.5 & Yes & 5 \\
\hline Kyon $^{\text {c }}$ & 4.0 & Yes & 4 \\
\hline Hofmann $^{\text {d }}$ & 3.5 & No & 4 \\
\hline Slocum Enterprises $^{\mathrm{e}}$ & 3.5 & No & 2 \\
\hline Biomedtrix $^{f}$ & 3.5 & No & 1 \\
\hline
\end{tabular}

${ }^{a}$ Synthes TPLO plate; DePuy Synthes Vet, West Chester, Pennsylvania, United States.

${ }^{b}$ Fixin TPLO plate; Intrauma S.r.I., Rivoli, Italy.

'Kyon TPLO plate; Kyon, Inc., Zurich, Switzerland.

${ }^{\mathrm{d} H}$ Hofmann TPLO plate; Hoffmann S.r.l, Monza, Italy.

eSlocum TPLO plate: Slocum Enterprises, Eugene, Oregon, United States (Company now closed).

fBiomedtrix multipurpose plate; BioMedtrix LLC, Whippany, New Jersey, United States. 
The difference between effective and planned changes in TPA was not significant ( $p$ 0.1080).

\section{Complications}

Intraoperative complications occurred in two cases. In one stifle, over-rotation that resulted in a TPA of $-8^{\circ}$ was immediately revised to produce a TPA of $5^{\circ}$. The second case involved a 8-year-old German Shepherd dog with a fissure of the lateral tibial cortex, which happened during power drill insertion of a locking screw. This was managed successfully by placing an additional $2.7 \mathrm{~mm}$ lag screw and a 2.7 locking compression plate on the craniolateral aspect of the tibia.

\section{Bone Healing}

All osteotomies healed uneventfully. Median bone healing score at the first radiographic re-evaluation was $4(2-4)$. The case that required adjunctive fixation because of a fissure of the lateral tibial cortex was the only one that had a healing score below 3 (2) at the 8-week radiographic re-evaluation, but at 3 months postoperatively, complete bone healing was evident radiographically.

\section{Discussion}

The exact biomechanical and clinical effects of proximal tibial deformities on contact and loading forces in the canine stifle joint are unknown. However, it has been shown that experimentally induced valgus deformity of the proximal tibia in Beagles leads to the development of osteoarthritis. ${ }^{5}$ This suggests that abnormal alignment at the stifle attributable to proximal tibial valgus deformity creates abnormal contact stresses and results in articular cartilage degeneration independent of cranial cruciate ligament rupture. ${ }^{5}$ Similarly, in a dog that underwent TPLO and varus-producing corrective tibial osteotomy in a staged procedure, it was hypothesized that tibia valga combined with cranial cruciate ligament rupture leads to lateral compartment osteoarthritis. ${ }^{6}$ In humans, it has been demonstrated that a valgus malalignment of the proximal tibia greater than $5^{\circ}$ after fixation of tibial fractures predisposes to early development of osteoarthritis. ${ }^{18}$ Although the biomechanical effects of tibia valga (and subsequent correction thereof) on contact and loading patterns in the canine stifle have not been defined, it can be presumed that when this condition occurs concurrently with cranial cruciate ligament deficiency, it should be treated. The technique of combining transverse tibial osteotomy with TPLO described previously proved to be effective in correcting the tibial deformity and can resolve torsional deformities as well. ${ }^{4}$ Nevertheless, this technique appears to be complex and requires additional double tension band wire, which may lead to prolonged surgical time and increased infection risk or failure of the fixation.

Our results showed that combining tibial plateau levelling and correction of proximal tibial valgus through a single crescentic closing wedge osteotomy in dogs with concurrent cranial cruciate ligament rupture and tibial deformity was successful without the need for additional implants. Our complication rate was low with only two intraoperative complications (5\%) and no postoperative complications.
All osteotomies except one were stabilized with conventional locking or non-locking TPLO plates from various manufacturers without additional implants. Bone healing with no implant-related complications was complete at the first radiographic follow-up 8 to 10 weeks postoperatively, and the median bone healing score was 4 . The use of standard plates designed for TPLO but supplied by various manufacturers, used without any additional implants, underlined that the inherent stability of the crescentic closing wedge was comparable to that of a standard TPLO. Standard healing times for regular TPLO have not been clearly defined and thus comparison of results is difficult, but our mean healing score at 8 to 10 weeks postoperatively was comparable with the findings of a study that investigated the efficacy of an anatomically contoured locking TPLO plate. ${ }^{17}$ The fissure of the lateral tibial cortex developed during power insertion of a locking screw in the shaft of the plate in one case was not considered to be related to the technique per se, but rather to insertion of the screw at a wrong angle and to the use of a power drill instead of a manual torque screwdriver.

We arbitrarily elected to use this technique in dogs with a value of greater than $97^{\circ}$ for the MMPTA, which is higher than the reference value ${ }^{16}$, and considered dogs with values less than $97^{\circ}$ candidates for standard TPLO rather than a more complicated operation. Further studies should be performed to determine which is the degree of deformity that requires correction. Also excluded were dogs with tibial torsion estimated radiographically to be greater than $10^{\circ} .{ }^{19}$ In those dogs, the combination of TPLO and transverse tibial osteotomy was considered more appropriate, but evaluation of torsion via computed tomography would have been more accurate. ${ }^{20,21}$

Overall, the technique resulted in precise correction of the deformity with an average postoperative mMPTA of $92.8^{\circ}$ (range: $88^{\circ}-97^{\circ}$ ), which was very close to the reference value of $93.30^{\circ} \pm 1.781^{\circ}$ reported by others. ${ }^{16}$ The difference between the planned and effective correction angles was not significant. The mMDTA angle did not differ significantly between preoperative and postoperative measurements, indicating that the surgical procedure had no effect on alignment of the distal tibia. Levelling of the tibial plateau was also considered successful based on an average postoperative TPA of $6.5^{\circ}$, which was close to the recommended value of $6^{\circ}$, and on the absence of a significant difference between the planned and effective correction angles. ${ }^{14,15}$

The presence of valgus deformity of the proximal tibia makes correct radiographic positioning in the mediolateral view difficult. It has been shown that radiographic malpositioning has an effect on TPA measurement and therefore we presumed that the initial over-rotation of the tibial plateau in one stifle was caused by miscalculation of the preoperative TPA. ${ }^{12}$ This underlines the importance of proper positioning for the preoperative mediolateral view, and the authors recommend the use of fluoroscopy to ensure that positioning includes superimposition of the femoral condyles. Once proper positioning was ensured, radiographic planning was effective in obtaining accurate postoperative tibial alignment.

Although the majority of our cases were medium-sized to large dogs, the technique was also feasible in small dogs. 
A limitation of the study was that the MMPTA was measured using the conventional method described by Dismukes and colleagues because it was the only one validated at the time of our first cases. ${ }^{16}$ The tangential method described by other authors has been shown to be more accurate. ${ }^{22}$ A further limitation was that it focused only on the alignment of the proximal tibia in the sagittal and frontal planes and did not evaluate the clinical outcome. Further studies are needed to evaluate the efficacy of the technique with regard to resolution of lameness and the progression of osteoarthritis in the long term.

\section{Conclusions}

The TPLO/MCCWO is an effective treatment for dogs with cranial cruciate ligament rupture and tibia valga. Accurate preoperative planning allowed correction of the tibial deformity in the frontal plane in combination with levelling of the tibial plateau.

\section{Authors' Contributions}

Luca Vezzoni and Aldo Vezzoni contributed to conception of study, study design, acquisition of data and data analysis and interpretation. Silvia Boiocchi contributed to acquisition of data. Bazzo Sara contributed to conception of study, study design and acquisition of data. All authors drafted, revised and approved the submitted manuscript.

\section{Conflict of Interest}

None declared.

\section{References}

1 Slocum B, Slocum TD. Tibial plateau leveling osteotomy for repair of cranial cruciate ligament rupture in the canine. Vet Clin North Am Small Anim Pract 1993;23(04):777-795

2 Krotscheck U, Nelson SA, Todhunter RJ, Stone M,Zhang Z. Long term functional outcome of tibial tuberosity advancement vs. tibial plateau levelling osteotomy and extracapsular repair in a heterogeneous population of dogs. Vet Surg 2016;45(02):261-268

3 Fitzpatrick N, Solano MA. Predictive variables for complications after TPLO with stifle inspection by arthrotomy in 1000 consecutive dogs. Vet Surg 2010;39(04):460-474

4 Weh JL, Kowaleski MP, Boudrieau RJ. Combination tibial plateau leveling osteotomy and transverse corrective osteotomy of the proximal tibia for the treatment of complex tibial deformities in 12 dogs. Vet Surg 2011;40(06):670-686

5 Panula HE, Helminen HJ, Kiviranta I. Slowly progressive osteoarthritis after tibial valgus osteotomy in young beagle dogs. Clin Orthop Relat Res 1997;(343):192-202

6 Petersen SW. Lateral compartment syndrome in the canine stifle Case report. Proceedings of the 34th Veterinary Orthopedic Society Annual Conference, Sun Valley, ID, March 3-10, 2007, 46pp
7 Mullaji AB, Marawar SV, Luthra M. Tibial articular cartilage wear in varus osteoarthritic knees: correlation with anterior cruciate ligament integrity and severity of deformity. J Arthroplasty 2008; 23(01):128-135

8 Coventry MB. Proximal tibial varus osteotomy for osteoarthritis of the lateral compartment of the knee. J Bone Joint Surg Am 1987; 69(01):32-38

9 Fitzpatrick N, Johnson J, Hayashi K, Girling S, Yeadon R. Tibial plateau leveling and medial opening crescentic osteotomy for treatment of cranial cruciate ligament rupture in dogs with tibia vara. Vet Surg 2010;39(04):444-453

10 Cook JL, Evans R, Conzemius MG, et al. Proposed definitions and criteria for reporting time frame, outcome, and complications for clinical orthopedic studies in veterinary medicine. Vet Surg 2010; 39(08):905-908

11 Caylor KB, Zumpano CA, Evans LM, Moore RW. Intra- and interobserver measurement variability of tibial plateau slope from lateral radiographs in dogs. J Am Anim Hosp Assoc 2001;37(03):263-268

12 Reif U, Dejardin LM, Probst CW, DeCamp CE, Flo GL, Johnson AL. Influence of limb positioning and measurement method on the magnitude of the tibial plateau angle. Vet Surg 2004;33(04): 368-375

13 Dismukes DI, Tomlinson JL, Fox DB, Cook JL, Witsberger TH. Radiographic measurement of canine tibial angles in the sagittal plane. Vet Surg 2008;37(03):300-305

14 Reif U, Hulse DA, Hauptman JG. Effect of tibial plateau leveling on stability of the canine cranial cruciate-deficient stifle joint: an in vitro study. Vet Surg 2002;31(02):147-154

15 Warzee CC, Dejardin LM, Arnoczky SP, Perry RL. Effect of tibial plateau leveling on cranial and caudal tibial thrusts in canine cranial cruciate-deficient stifles: an in vitro experimental study. Vet Surg 2001;30(03):278-286

16 Dismukes DI, Tomlinson JL, Fox DB, Cook JL, Song KJ. Radiographic measurement of the proximal and distal mechanical joint angles in the canine tibia. Vet Surg 2007;36(07):699-704

17 Kowaleski MP, Boudrieau RJ, Beale BS, Piras A, Hulse D, Johnson KA. Radiographic outcome and complications of tibial plateau leveling osteotomy stabilized with an anatomically contoured locking bone plate. Vet Surg 2013;42(07):847-852

18 Parkkinen M, Madanat R, Mustonen A, Koskinen SK, Paavola M, Lindahl J. Factors predicting the development of early osteoarthritis following lateral tibial plateau fractures: mid-term clinical and radiographic outcomes of 73 operatively treated patients. Scand J Surg 2014;103(04):256-262

19 Petazzoni M. Radiographic measurements of the tibia. In: Petazzoni M, Jaerger GH, eds. Atlas of Clinical Goniometry and Radiographic Measurements of the Canine Pelvic Limb. Duluth, GA: Merial; 2008:60-69

20 Apelt D, Kowaleski MP, Dyce J. Comparison of computed tomographic and standard radiographic determination of tibial torsion in the dog. Vet Surg 2005;34(05):457-462

21 Barnes DM, Anderson AA, Frost C, Barnes J. Repeatability and reproducibility of measurements of femoral and tibial alignment using computed tomography multiplanar reconstructions. Vet Surg 2015;44(01):85-93

22 Lambert RJ, Wendelburg KL. Determination of the mechanical medial proximal tibial angle using a tangential radiographic technique. Vet Surg 2010;39(02):181-186 